\title{
Mapas de riesgo para Hantavirus en el Parque Nacional Conguillío, sur de Chile
}

\author{
Hantavirus risk maps for Conguillío National Park, southern Chile
}

\author{
ANDRÉS MUÑOZ-PEDREROS ${ }^{1, *}$, PATRICIO RUTHERFORD ${ }^{2} \&$ CLAUDIA GIL ${ }^{2}$ \\ ${ }^{1}$ Escuela de Ciencias Ambientales, Facultad de Recursos Naturales, Universidad Católica de Temuco, Temuco, Chile \\ ${ }^{2}$ Centro de Estudios Agrarios y Ambientales, Casilla 15-D, Valdivia, Chile; \\ * e-mail para correspondencia: amunoz@uct.cl
}

\begin{abstract}
RESUMEN
El riesgo de infección con Hantavirus depende de factores, que determinan una probabilidad de contagio con los reservorios, tales como: (a) la estructura vegetacional y el uso del suelo como un escenario primario, donde los factores específicos de composición, estructura y densidad de la vegetación detallan elementos relacionados con el hábitat de los reservorios. (b) La existencia de poblaciones de roedores reservorio. (c) Establecimientos humanos, como disponibilidad y densidad de caminos, áreas habitadas o de presencia humana (e.g., casas, bodegas). Estos tres factores, hábitat (probabilidad de ocurrencia del reservorio), roedores seropositivos (peligro de contagio) y humanos (población expuesta), conjugados, proporcionan los elementos de juicio necesarios para establecer el riesgo. Es importante considerar que estos factores tienen una dinámica de cambio estacional durante el año y modificaciones ambientales naturales y antrópicas. Así, buscamos comprender el riesgo a que está sometido el ser humano en el espacio rural. Los modelos espaciales corresponden a representaciones de la realidad observada en un área determinada y condicionada a diversos factores geográficos, topográficos, biológicos, climáticos, etc. El objetivo de este estudio fue establecer sectores potenciales de riesgo al hantavirus en un parque nacional de la IX Región de Chile empleando cartas temáticas de variables ambientales en un Sistema de Información Geográfica para analizar fotografías aéreas mediante fotointerpretación, transferencia, digitalización y manejo de base de datos gráfica y alfanumérica, validada en terreno. La capa vectorial fue "rasterizada" con un tamaño de píxel de $50 \mathrm{~m}$. El mapa de riesgo se construyó utilizando un modelo aditivo de capas mediante el uso del software Model Builder 1.0, extensión de ArcView 3.2. La base del procedimiento fue el proceso de "overlay" aritmético, que sobrepone las capas adicionando los términos de valor de cada variable utilizando la misma de ponderación en cada una de ellas (e.g., uso del suelo, hábitat del reservorio, seropositividad del reservorio, casos y asentamientos humanos). Presentamos un mapa de riesgo que señala como principales áreas de riesgo, precisamente donde se emplaza la infraestructura turística principal de este visitado parque.
\end{abstract}

Palabras clave: Hantavirus, mapas de riesgo, sur de Chile.

\begin{abstract}
The risk of infection with Hantavirus depends on factors that determine a probability of contagion with the reservoirs: (a) the vegetation structure and the land use as a primary scene, where specific factors such as composition, structure and density of the vegetation describe elements related to the habitat of the reservoirs. (b) The existence of populations of reservoir rodents. (c) Human establishments, such as availability and density of roads, inhabited areas or human presence (e.g., houses, warehouses). These three factors, brought together, provide the necessary facts to establish the risk. It is important to consider that these factors have a dynamics of seasonal change during the year and natural and man-made environmental modifications. In this way, we seek to understand the risk to which humans beings are submitted in the rural space. The spatial models correspond to representations of the reality observed in a certain area and determined to diverse geographical, topographic, biological, climatic factors, etc. The aim of this study was to establish potential sectors of risk to Hantavirus in a national park of the Region IX of Chile using thematic maps of environmental variables in a Geographical Information System to analyze aereal photograhs by means of photo interpretation, transference, digitalization and graphical-alphanumerical database managing. The vector layer was rasterized using a pixel size of $50 \mathrm{~m}$. The map of risk was constructed using an additive model of layers through the Model Builder 1.0 software, an extension of ArcView 3.2. The base of the procedure was the arithmetic overlay process what overlaps the layers adding the numeric terms of each variable using the
\end{abstract}


same ponderation for every layer (e.g., land use, habitat for the reservoir, seropositivity of the reservoir, human cases and instalations). We present a risk map, for this highly visited park, which signal as the most important risk areas, the areas where all of the turistic infrastructure is installed.

Key words: Hantavirus, maps of risk, south of Chile.

\section{INTRODUCCIÓN}

En 1976, en Corea, se aisló por primera vez el agente causal de la fiebre hemorrágica, denominándose a este nuevo género Hantavirus y clasificándolo en la familia Bunyaviridae. Estos virus causan enfermedades zoonóticas que se asocian estrechamente a los roedores de la familia Muridae y en América a los roedores de la subfamilia Sigmodontinae. Al parecer, existiría coevolución de virus y roedores reservorios de manera que, usualmente, cada virus está asociado a una especie de roedor (Schmaljohn \& Hjelle 1997, Spotorno et al. 2000). En Chile, el serotipo Andes causa un síndrome hemorrágico pulmonar (SHP), habitualmente grave y que puede ser mortal. La puerta de entrada para el hantavirus la constituye, principalmente, el epitelio de las vías respiratorias. Luego de una fase inicial de replicación en el sistema respiratorio, el virus se disemina vía sanguínea al resto del organismo, causando una infección generalizada, con compromiso de los diferentes órganos y musculatura (Tapia 1997, Sotomayor \& Aguilera 2000). El punto crítico es sobrevivir al colapso cardiorrespiratorio producto del paso de plasma a los alvéolos, por lo que el síndrome se ha redefinido como síndrome cardiopulmonar por Hantavirus (SCPH) (Castillo \& Ossa 2002).

En las enfermedades transmisibles existen tres elementos básicos: el reservorio, que corresponde al substrato donde los gérmenes patógenos habitan, se multiplican y se mantienen en la naturaleza (e.g., roedores); las vías de transmisión, que es el mecanismo ocupado por el germen para pasar a un huésped (e.g., el aire, vectores, contacto directo), y el huésped susceptible, que son los organismos que reúnen las condiciones para que el germen patógeno se desarrolle y produzca la enfermedad. Para comprender la ocurrencia de contagio se deben considerar dos grandes variables: la ecología de los reservorios, en este caso los roedores y la sociología de los seres humanos.
Respecto a los reservorios, los roedores pertenecen al orden Rodentia (Bowdich 1821) y se distribuyen en casi todo el planeta, constituyendo sobre el $40 \%$ de todas las especies de la clase Mammalia (De Blaise \& Martin 1981). Su extraordinaria plasticidad les ha llevado a ocupar los más diversos hábitats, adaptándose tanto a los ambientes terrestres (arbóreo, superficial y subterráneo), como semiacuáticos. En Chile este orden está representado por 65 especies, de las cuales 60 son nativas y cinco introducidas (Yáñez \& Muñoz-Pedreros 2000). Se considera a la lauchita de los espinos (lauchita colilarga) (Oligoryzomys longicaudatus (Bennett, 1832) como el reservorio del virus Andes, pero se discute el rol de las otras especies de roedores silvestres que se han registrado como seropositivas en Chile: laucha olivácea (Abrothrix olivaceus (Waterhouse, 1837), laucha de pelo largo (Abrothrix longipilis (Waterhouse, 1837), laucha de Sanborn (Abrothrix sanborni (Osgood, 1943), lauchón orejudo de Darwin (Phyllotis darwini (Waterhouse, 1837) y un caso en el pericote austral (Loxodontomys micropus (Waterhouse, 1837). Para más antecedentes véase Toro et al. (1998), Mills (1999), Pavletic (2000a), Galeno et al. (2000) y Ortiz et al. (2004).

La manera principal en que el hantavirus se trasmite a los seres humanos es al respirar el aire contaminado con saliva, orina o heces del reservorio (Sotomayor \& Aguilera 2000). Puede también ser transmitida por manipulación o mordeduras de roedores o de persona a persona (Padula et al. 1998). No hay evidencia que los gatos o perros transmitan la enfermedad a los seres humanos.

El contacto del ser humano con roedores portadores del virus afecta a las personas vinculadas, ya sea por trabajo, deporte, turismo o esparcimiento, con áreas rurales que poseen poblaciones de reservorios, y en alta proporción adquieren la enfermedad en sus lugares de residencia (Sotomayor \& Aguilera 2000). Pese a que una proporción menor de los casos registrados en Chile son visitantes a los 
espacios rurales $(<10 \%)$ por lo masivo de la concurrencia de turistas a las áreas silvestres, pueden ser una importante población eventualmente expuesta a riesgo de infección (> 140.000 visitas solo en parques nacionales). El riesgo de infección con Hantavirus depende de variados factores que determinan una probabilidad de contagio con los reservorios. De este modo, un primer elemento a considerar es la estructura vegetacional y el uso del suelo como un escenario primario, donde los factores específicos de composición, estructura y densidad de la vegetación detallan elementos relacionados con el hábitat de los reservorios. Este elemento se relaciona con la probabilidad de ocurrencia del reservorio en un área determinada. Un segundo elemento es la existencia probada de poblaciones de roedores reservorio, esto mediante muestreos en terreno que incluyen la toma de muestras de sangre necesarias para establecer la seropositividad de los ejemplares analizados. Este elemento establece el peligro de contagio de la enfermedad. Un tercer elemento son los establecimientos humanos, como disponibilidad y densidad de caminos, áreas habitadas o de presencia humana (casas, bodegas), véase Pavletic (2000b). Este elemento establece la exposición de las poblaciones humanas al peligro. Estos tres factores, conjugados, proporcionan los elementos necesarios para establecer el riesgo (peligro + exposición). Es importante considerar que estos factores tienen una dinámica de cambio estacional durante el año y modificaciones en períodos más largos, como se detallará más adelante.

De este modo, buscamos un modelo espacial que ayude a comprender el riesgo al que está sometido el ser humano en el espacio rural. Estos modelos corresponden a representaciones de la realidad observada en un área determinada y condicionada a diversos factores geográficos, topográficos, biológicos, climáticos y otros. El propósito de este modelo es simplemente ayudar en la comprensión de fenómenos cuyo resultado se debe a la interacción de muchos factores como los mencionados anteriormente, describiendo o prediciendo ese resultado en el mundo real. Existen estudios en este sentido pero a una resolución espacial no siempre óptima para orientar medidas efectivas de prevención y control de los reservorios (e.g., Glass et al. 2002, Sánchez-Cordero et al. 2005).
El objetivo de este estudio es establecer sectores potenciales de riesgo al Hantavirus en el Parque Nacional Conguillío, en el sur de Chile, empleando cartas temáticas de variables ambientales en un Sistema de Información Geográfico (SIG) y proponer un procedimiento con capacidad predictiva del riesgo a Hantavirus.

\section{MATERIALES Y MÉTODOS}

\section{Área de estudio}

El estudio se desarrolló en los laboratorios de Geomática y de Manejo de Ecosistemas del Centro de Estudios Agrarios y Ambientales, y la metodología generada se sometió a prueba en el Parque Nacional Conguillío, área silvestre protegida de 60.833 ha totales ubicada en las comunas de Melipeuco, Vilcún, Curacautín, Lonquimay (IX Región administrativa de Chile) caracterizada por la presencia del volcán Llaima como principal agente modelador del paisaje. Este parque, junto a la Reserva Nacional Alto Biobío constituyen la Reserva Mundial de la Biosfera Araucarias (Benoit 2004). En su interior se conserva una diversidad de ecosistemas en donde se destacan los ambientes de humedales (e.g., lagunas), altas cumbres, bosques y escoriales. Es relevante la presencia de importantes bosques cordilleranos de araucaria (Araucaria araucana (Mol.) K. Koch) que se entremezclan con especies de Nothofagus como coihue (Nothofagus dombeyi (Mirbel) Oerst.), raulí (Nothofagus alpina (P. et E.) Oerst.), lenga (Nothofagus pumilio Kasser) y ñirre (Nothofagus antarctica (G. Forster) Oerst.). Es importante mencionar el fenómeno de sucesión vegetacional con plantas colonizadoras como cola de zorro (Setaria sp.), ciprés de cordillera (Austrocedrus chilensis (D. Don) Pic. Ser. Et Bizz.) y chauras (Pernettya sp.) que surgen en suelos de origen volcánico.

El área de estudio (383' S, 71³8' O) corresponde a 18.259 ha del Parque Nacional Conguillío a unos $20 \mathrm{~km}$ desde la localidad de Melipeuco hacia el norte, a través del camino que bordea el río Allipén y a unos $1.100 \mathrm{~m}$ de altitud. Esta área corresponde a sitios en los que se encuentran principalmente formaciones dominadas por A. araucana, en mezcla con 
otras especies como $N$. dombeyi, $N$. antarctica y $N$. pumilio, las cuales varían según la altitud sobre el nivel del mar, posición topográfica y condiciones edáficas.

\section{Cartografía}

Se realizó un vuelo fotogramétrico para obtener fotografías aéreas en colores a escala aproximada 1:10.000. Estas fotografías fueron fotointerpretadas utilizando un estereoscopio de espejos Wild ST. El criterio de rodalización de las categorías vegetacionales respondió a las características de tono, estructura, cobertura y posición geográfica. Se utilizó una unidad mínima cartografiable de 1 ha y ancho mínimo de polígono de $25 \mathrm{~m}$, características óptimas considerando la escala de toma de las fotografías aéreas y necesarias para los posteriores procesos de rasterización y "overlay". La rasterización es la transformación de un conjunto de datos vectoriales, formado por líneas, puntos o polígonos, en una estructura de datos raster, representándose, así, en una matriz de pequeñas celdas cuadradas de igual tamaño, cada una con un valor numérico codificado en su interior. El "overlay" superpone fuentes de información distintas por lo que va asociado con las funciones de cruce de información vectorial (intersección de capas), fundamental en los Sistemas de Información Geográfica (SIG).

Posterior a la fotointerpretación se realizó la transferencia de las áreas efectivas de los pares estereoscópicos mediante un Sketch-Master Leica a escala base 1:15.000. La transferencia fue digitalizada en una mesa Numonics AccuGrid III mediante el software ArcInfo 4. La capa digital resultante fue transformada a formato shapefile para permitir el manejo en ArcView, donde fue asociada la base de datos anexa. La capa vectorial fue rasterizada con un tamaño de píxel de $50 \mathrm{~m}$. Todo este procedimiento contó con apoyo cartográfico de manera de prever las características de topografía y vegetación que fueron interpretadas. Se recopilaron las respectivas cartas regulares IGM en escala 1:50.000 y la cartografía vegetacional del Proyecto Catastro de Bosque Nativo (CONAF-CONAMA 1999). La fotointerpretación se realizó no solo desde el punto de vista vegetacional, sino que procurando un énfasis sobre los distintos ambientes en los cuales fue posible encontrar los roedores portadores de Hantavirus. Para ello se consideraron los siguientes aspectos: uso de suelo (tipo de vegetación), cobertura (densidad), estructura (estado sucesional del bosque nativo), altura de la vegetación y localización topográfica.

Paralelamente a las actividades con el material fotográfico, se desarrollaron personalizaciones (simplificación de funciones para el usuario) del software ArcView 3.2 para las presentaciones de los mapas de riesgo utilizando el lenguaje AVENUE. De este modo, se elaboraron aplicaciones para el despliegue de coberturas, presentación de las intersecciones geográficas y presentación de resultados. Esta personalización permitió simplificar el software original, facilitando la transferencia de la tecnología SIG hacia el usuario.

\section{Mapa de riesgo}

Los mapas de riesgo se construyeron utilizando un modelo de "overlay" aritmético simple utilizando el software Model Builder 1.0, una extensión de ArcView y su módulo Spatial Analyst. Esto permitió que cada cobertura considerada en el modelo se constituya en un factor, que reunidos expresaron la probabilidad de riesgo frente al Hantavirus. Las cartas empleadas (ingresadas como coberturas espaciales) fueron: carta de uso del suelo, carta de hábitat del reservorio, carta de seropositividad y carta de asentamientos humanos, las que superpuestas permitieron generar la carta de riesgo.

Para la carta de uso del suelo se procedió a una descripción de terreno de las unidades cartográficas homogéneas, delimitadas previamente en el proceso de fotointerpretación. Del mismo modo, se verificó la ubicación geográfica del área de estudio seleccionada, de manera de especificar su ubicación dentro de la información cartográfica. Con esta primera visión general se procedió a emplear las fotografías aéreas para elaborar las cartas bases del área de estudio en una escala 1:15.000. Estas cartas aportaron la siguiente información: curvas de nivel, red hidrográfica, caminos y senderos, áreas de uso humano (casas, cabañas, áreas de camping, áreas de picnic, senderos), puntos de muestreo, grilla con coordenadas. Sobre esta carta base se agregó información sobre uso del suelo. 
La carta de hábitat del reservorio correspondió a la especificación del hábitat del reservorio, entregando esta caracterización la probabilidad de encuentro de las poblaciones de roedores reservorios. Oligoryzomys longicaudatus estará presente en un hábitat determinado en la medida que exista alimento y refugio necesario para cubrir sus necesidades. Una primera aproximación fue emplear la carta de uso del suelo y seleccionar de ella los hábitats probables de ser ocupados por el reservorio, realizando un análisis visual de las unidades cartográficas homogéneas en la base de datos, considerando estructura de la vegetación, cobertura y composición detallada según especies dominantes. En el área de estudio fueron: asociaciones boscosas, matorrales y praderas. La segunda aproximación fue caracterizar los distintos microhábitats, presentes en las áreas de estudio, lo que se logró con dos procedimientos complementarios: (a) estudio de la composición vegetacional, realizado mediante la técnica del punto cuadrado (Cottam et al. 1953), que pondera la vegetación registrada a nivel de microhábitat. La sistemática y nombres librescos siguieron a Marticorena \& Quezada (1985) y Hoffmann (1982), respectivamente. (b) Estudio de la estructura vegetacional, analizando las variables de los estratos herbáceos y arbustivos, además de perfiles de densidad de follaje. Se empleó la metodología descrita por MacArthur \& MacArthur (1961). Esto permitió caracterizar el hábitat para la especie reservorio.

La carta de seropositividad se construyó a partir de información sobre las poblaciones de roedores, obtenida mediante la instalación de grillas y redes con trampas Sherman plegables cebadas con avena machacada en el área de estudio. Se efectuaron muestreos de cinco noches con revisión diaria con las medidas de bioseguridad recomendadas por CDS/NIH/ OPS/OMS (s/f). Los animales capturados fueron sometidos al marcaje, registro morfométrico de rutina (De Blaise \& Martin 1979). La nomenclatura siguió a Yáñez \& Muñoz-Pedreros (2000). Se estableció la abundancia específica de los roedores capturados.

En el área de estudio se identificaron seis estaciones de muestreo, en ambientes de diferentes características, los que se denominaron: Los Carpinteros (bosque mixto de
A. araucana y $N$. dombeyi), Centro de Informaciones (bosque de A. araucana con matorral denso), Cabañas Hut (vegetación abierta dominada por A. araucana), Arco Iris/ Turbina (bosque de Nothofagus spp), Captrén (bosque de A. araucana, N. pumilio, Pernettya sp y $C$. culeu, con sotobosque ralo) y Escorial junto al lago Conguillío (escoria volcánica con gramíneas ralas). Se realizaron seis muestreos de terreno en enero de 1999, abril de 2000, enero de 2001, abril de 2001, noviembre de 2001 y marzo de 2002. Las muestras de sangre de los roedores reservorios fueron obtenidas mediante punción retrorbital o punción intracardíaca, las que se depositaron en criotubos y fueron congeladas en nitrógeno líquido, para ser enviadas al laboratorio de bioseguridad de la Universidad Austral de Chile, donde se obtuvo la seropositividad a Hantavirus mediante "positive enzyme-linked immunosorbent assay" IgG, ELISA (Elgh et al. 1997).

Los resultados fueron incorporados para cada sitio de muestreo, construyéndose la carta con los puntos de captura georreferenciados de los ejemplares seropositivos. En el caso de $O$. longicaudatus se consideró su ámbito de hogar (Muñoz-Pedreros et al. 1990) como el área de influencia del roedor. En Concepción presentó ámbitos de hogar con áreas máximas en verano $\left(3.384 \mathrm{~m}^{2}\right)$ y mínimas en otoño $\left(677 \mathrm{~m}^{2}\right)$. En Valdivia presentó una gran fluctuación en el ámbito de hogar (320 a $4.800 \mathrm{~m}^{2}$ ). En la provincia de Neuquén, Argentina, presentó un ámbito de hogar con una superficie media de $918,8 \mathrm{~m}^{2}$, con valores significativamente mayores en los machos adultos (valor medio: $1.007 \mathrm{~m}^{2}$ ) que en las hembras adultas (valor medio: 680,8 $\mathrm{m}^{2}$ ). La superficie menor registrada es de $320 \mathrm{~m}^{2}$ y la más extensa de $4.800 \mathrm{~m}^{2}$, por lo que se consideró, con un margen de seguridad, en 0,5 hectárea la superficie con presencia probable del reservorio. Asumiendo áreas de influencia concéntricas se establecen dos niveles de presencia probable de reservorios seropositivos, la primera, de probabilidad alta, en un anillo de 0,5 ha y la segunda un área de probabilidad media de 1,5 ha, con la posición de la georreferencia del ejemplar seropositivo como centro.

La carta de asentamientos humanos se construyó a partir de la digitalización de la información de artificios entregada desde las fotografías aéreas y verificación en terreno, 
identificándose las construcciones humanas (e.g., casas, bodegas, galpones). Se determinó un área de influencia directa de $100 \mathrm{~m}$ de radio y para el caso de los caminos secundarios y huellas, se les asignaron $30 \mathrm{~m}$ de borde. Posteriormente, se unieron la red de caminos y los puntos de construcciones. Esto constituyó la zona de exposición de humanos en el área de estudio.

Finalmente, la carta de riesgo se construyó superponiendo las cartas ya descritas mediante el uso de Model Builder 1.0. El procedimiento consistió en vincular las bases de datos que sostienen cada carta. Los mapas de riesgo se crearon en base a un modelo aritmético sujeto a probabilidad, lo que se ha traducido en la utilización de herramientas multicriterio para obtener el objetivo final. Básicamente, esta técnica integró las operaciones de los SIG con técnicas de ayuda a la decisión, incrementándose así la potencia operativa de la información territorial recolectada a partir de diferentes fuentes, como otra información cartográfica, u otras capas ("layers") de información territorial geográfica y biológica. De este modo, se integró la totalidad de las capas necesarias para la generación de modelos, en este caso de riesgo, que fueron obtenidas como producto final. Esta representación geográfica permitió confeccionar las capas y variables que se muestran en la Tabla 1 y Fig. 1.

\section{RESULTADOS Y DISCUSIÓN}

\section{Carta Base, hábitat y seropositividad}

La Carta Base del área de estudio reveló que las instalaciones humanas se presentan concentradas y cercanas a los cuerpos de agua laguna Conguillío, laguna Captrén y laguna Arcoiris. En la Tabla 2 se muestra el uso del suelo en una superficie total de $18.259,8$ hectáreas. Predominan, claramente, el bosque nativo $(52,8$ $\%)$ y las áreas desprovistas de vegetación, principalmente escoria volcánica $(34,7 \%)$. Es notable constatar que los otros usos tienen representaciones menores al $6 \%$. En el uso del suelo disgregado se aprecia que los bosques de A. Araucana - N. Pumilio - Pernettya sp. - quila Chusquea culeu Kunth (18\%) y N. Dombeyi - N. Alpina - C. coleu $(13,5 \%)$ poseen la mayor representación.

\section{Selección y caracterización de hábitats repre- sentativos}

El sector Los Carpinteros corresponde a un bosque mixto de A. araucana y $N$. dombeyi, especies componentes del dosel dominante y codominante, observándose regeneración en claros de ambas especies aunque con mayor éxito por parte de la conífera. El sotobosque se

TABLA 1

Variables consideradas para elaborar cartas de riesgo a Hantavirus en el Parque Nacional Conguillío, sur de Chile

Variables considered in the creation of Hantavirus risk maps of Conguillío National Park, southern Chile

\begin{tabular}{ll}
\hline Variable & Concepto implícito \\
\hline Altitud & Distribución \\
Macrohábitat & Presencia general \\
Microhábitat & Presencia específica \\
Caminos y senderos & Área de contacto \\
Construcciones y camping & Área de contacto \\
Actividades antrópicas & Área de contacto \\
Casos humanos registrados & Registro histórico/probabilístico \\
Frecuencia de reservorios & Registro histórico/probabilístico \\
Abundancia del reservorio & Registro histórico/probabilístico \\
Seropositividad del reservorio & Registro histórico/probabilístico
\end{tabular}




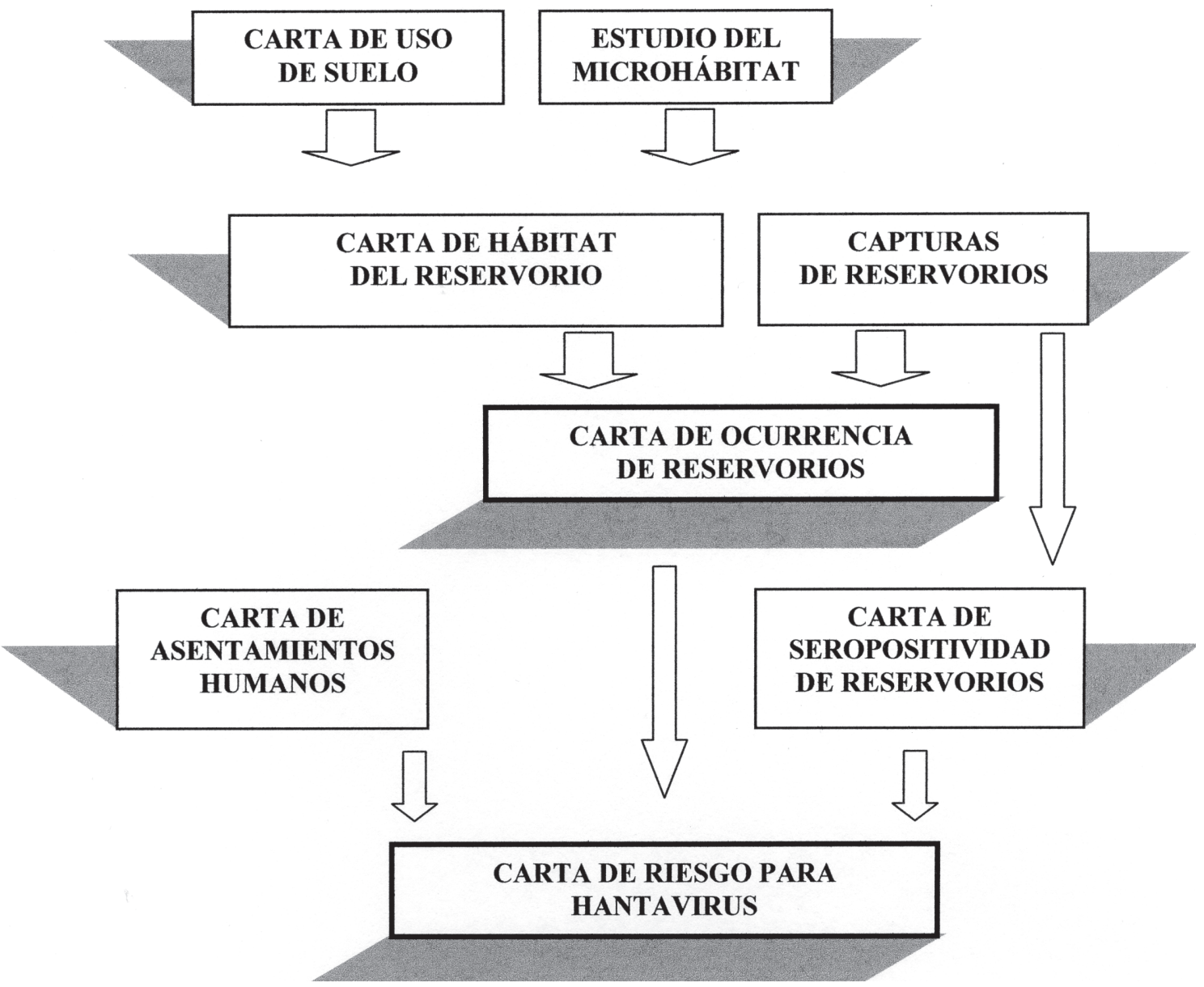

Fig. 1: Diagrama de superposición de cartas para la construcción de cartas de riesgo para Hantavirus. Diagram of Hantavirus risk map overlay.

encuentra dominado principalmente por $C$. culeu, siendo importante también la presencia de canelo (Drimys winteri J.R. et G. Forster), maitén de Magallanes (Maitenus magellanica (Lam.) Hook.), michay (Berberis buxifolia Lam.) y taique (Desfontainea spinosa $\mathrm{R}$. et $\mathrm{P}$ ).

El sector Centro de Informaciones corresponde a un bosque dominado por $A$. araucana en el dosel dominante, aunque presentándose en baja cobertura, existiendo un matorral denso de N. antarctica, Chusquea sp., P. pumila (L.f.) Hook.) y brecillo (Empetrum rubrum Vahl ex Willd.) que se encuentra en alta densidad bajo los 0,5 $\mathrm{m}$ de altura.

El sector Cabañas Hut corresponde a un área de vegetación abierta, sobre escoria, dominada por A. araucana en el dosel superior. El estrato bajo está compuesto por $N$. antarctica, Festuca scabriuscula Phil., Poa sp.,
Pernettya sp. y Chusquea cumingii Nees. La vegetación se desarrolla en manchones localizados sobre la escoria volcánica, siendo de fácil transitabilidad.

El sector correspondiente a Arcoiris/Turbina corresponde a un bosque dominado por $N$. dombeyi, con roble Nothofagus obliqua (Mirbel) y $N$. alpina cercano a un curso de agua. Existe una fuerte presencia de $C$. culeu en el sotobosque, con presencia de $D$. winteri, $B$. buxifolia y M. magellanica.

El sector Captrén es un bosque de $A$. araucana, N. pumilio, Pernettya sp. y C. culeu, con un sotobosque ralo.

El sector Escorial, junto al lago Conguillío, es un sector de escoria volcánica cubierto, en forma poco densa, por gramíneas y con ejemplares de $N$. antarctica dispuestos en forma muy rala. 
TABLA 2

Uso del suelo general en el área de estudio del Parque Nacional Conguillío, 2001

Land use in the study area, Conguillío National Park, southern Chile

\begin{tabular}{lll}
\hline Uso de suelo & $\begin{array}{l}\text { Superficie } \\
\text { (ha) }\end{array}$ & Porcentaje \\
\hline Pradera altoandina & 289,4 & 1,6 \\
Pradera con árboles aislados & 117,3 & 0,6 \\
Matorral con praderas & 4,5 & 0,0 \\
Matorral arborescente & 495,6 & 2,7 \\
Plantación forestal & 9,9 & 0,1 \\
Bosque nativo & $9.634,3$ & 52,8 \\
Humedal & 3,5 & 0,0 \\
Área sin vegetación & $6.345,2$ & 34,7 \\
Nieve & 146,1 & 0,8 \\
Cuerpos de agua & 926,2 & 5,1 \\
Total & $18.259,8$ & 100,0 \\
\hline
\end{tabular}

\section{Captura de reservorios}

En la Tabla 3 se muestran las capturas totales, 204 ejemplares (201 roedores y tres marsupiales), obtenidas en seis muestreos entre 1999 y 2002, en diferentes ambientes del Parque Nacional Conguillío. Para su contabilización se excluyeron las recapturas. Se aprecia que la especie más abundante fue Abrothrix longipilis $(63,2 \%)$, seguida de $A$. olivaceus $(19,1 \%)$ y de $O$. longicaudatus $(14,2$ $\%)$, proporción que tiende a mantenerse en todos los hábitats muestreados, aunque varían sus números. El hábitat de bosque de $A$. araucana - N. antarctica y Pernettya sp. (Centro de Información) y el bosque de $N$. dombeyi - N. obliqua - N. alpina - C. culeu (sector de Arcoiris/Turbina) acaparan las mayores capturas (96 y $64 \%$ respectivamente) y las más bajas son el bosque de A. araucana, $N$. pumilio, Pernettya sp y C. culeu (Captrén) y en el $N$. antarctica muy ralo con gramíneas (Escorial junto al lago Conguillío). Una situación intermedia muestran los otros dos hábitats, el bosque de $N$. dombeyi, N. pumilio, $A$. araucana y $C$. culeu (Los Carpinteros) y matorral de $N$. antarctica (Cabañas Hut).

Las poblaciones del reservorio fueron muy bajas en primavera-verano y más altas en otoño-invierno, lo que es concordante con lo descrito en otras localidades de la zona centro y sur de Chile (e.g., Muñoz-Pedreros 1992, Meserve et al. 1999).

\section{Hábitat}

Con la información reunida, el análisis de la carta base, de la carta de uso del suelo, tanto general como por agrupaciones vegetacionales, estudios en terreno de los diferentes microhábitats y de los registros de captura de los micromamíferos se pudieron agrupar los hábitats presentes en el Parque Nacional Conguillío en cinco tipos fundamentales, según sus diferentes probabilidades de encuentro (ocurrencia) de ejemplares de $O$. longicaudatus: (1) hábitat con baja probabilidad de ocurrencia del reservorio; (2) hábitat con media probabilidad de ocurrencia del reservorio; (3) hábitat con alta probabilidad de ocurrencia del reservorio; (4) hábitat con muy alta probabilidad de ocurrencia del reservorio; (5) hábitat no evaluado por muy baja ocurrencia humana y/o condiciones muy desfavorables para la ocurrencia de ejemplares del reservorio.

Fue importante la caracterización en terreno de los diferentes estratos vegetacionales. Considerando los requerimientos simultáneos del reservorio, tanto del estrato herbáceo (importante para la provisión de alimento) y el estrato arbustivo denso (refugio y alimento) y ciertos árboles (como proveedores de semillas), encontramos que el hábitat que cumplía estos requisitos ideales era el bosque de $A$. araucana - N. antarctica - Pernettya sp., así como el de $N$. dombeyi - N. obliqua - N. alpina - C. culeu. Por otro lado, fueron hábitats desfavorables, por ejemplo, el matorral de $N$. Antarctica y el de $N$. antarctica muy ralo con gramíneas. Esto coincide con las capturas presentadas en la Tabla 3.

En la Tabla 4 se muestra que la superficie con probabilidad de ocurrencia muy alta y alta se restringe a un $14,9 \%$ de la superficie estudiada, por lo que se concluye que las poblaciones de roedores estarían heterogéneamente distribuidas en el área de estudio. En la Fig. 2 se muestra la Carta de Hábitat probable para los roedores documentadamente seropositivos $(O$. longicaudatus, A. olivaceus, A. longipilis, L. micropus), tanto en este como en estudios previos. 


\section{Seropositividad}

En la Tabla 5 se muestra la seropositividad por especies registrada en el Parque Nacional Conguillío. La seropositividad total de roedores silvestres, sobre una muestra de 201 ejemplares fue de $4,0 \%$. Fueron positivos cuatro ejemplares de $O$. longicaudatus, con una seropositividad de
$13,8 \%$ (29 ejemplares muestreados) y cuatro ejemplares de $A$. olivaceus, con una seropositividad de 3,1 \% (129 ejemplares muestreados). Todos los casos se registraron en los sectores de Arcoiris (bosque de N. dombeyi N. obliqua - N. alpina - C. culeu) o Centro de Informaciones (bosque de A. araucana - $N$. antarctica y Pernettya sp.).

TABLA 3

Capturas totales y abundancia de micromamíferos capturados en el Parque Nacional Conguillío (IX Región de Chile) en seis muestreos entre 1999 y 2002

Total captures and abundance of small mammals captured in Conguillío National Park, during 6 sampling periods from 1999 to 2002

\begin{tabular}{|c|c|c|c|c|c|c|c|c|}
\hline \multirow[t]{2}{*}{ Especie } & \multicolumn{6}{|c|}{ Sector y hábitat } & \multirow[b]{2}{*}{ Total } & \multirow[b]{2}{*}{$\begin{array}{c}\text { Abundancia } \\
(\%)\end{array}$} \\
\hline & $\begin{array}{l}\text { Arcoiris } \\
\text { N. dombeyi, } \\
\text { N. oblicua, } \\
\text { C. alpina, } \\
\text { C. culeu }\end{array}$ & $\begin{array}{l}\text { Centro de } \\
\text { visitantes } \\
\text { A. arau- } \\
\text { cana, } \\
\text { N. antarc- } \\
\text { tica y } \\
\text { Pernettya } \\
\text { sp. }\end{array}$ & $\begin{array}{l}\text { Cabañas } \\
\text { Hut } \\
\text { N. antarc- } \\
\text { tica }\end{array}$ & $\begin{array}{l}\text { Los Carpin- } \\
\text { teros } \\
\text { N. dombeyi, } \\
\text { N. pumilio, } \\
\text { Pernettya } \\
\text { sp., C. } \\
\text { culeu }\end{array}$ & $\begin{array}{c}\text { Captrén } \\
\text { A. arau- } \\
\text { cana, } \\
\text { N. pumilio, } \\
\text { Pernettya } \\
\text { sp., C. } \\
\text { culeu }\end{array}$ & $\begin{array}{l}\text { Escorial } \\
\text { N. antarc- } \\
\text { tica (muy } \\
\text { ralo con } \\
\text { gramíneas) }\end{array}$ & & \\
\hline Oligoryzomys longicauda & atus 7 & 20 & 1 & 1 & 0 & 0 & 29 & 14,2 \\
\hline Abrothrix olivaceus & 10 & 18 & 5 & 1 & 4 & 1 & 39 & 19,1 \\
\hline Abrothrix longipilis & 41 & 55 & 7 & 14 & 4 & 8 & 129 & 63,2 \\
\hline Loxodontomis micropus & 1 & 2 & 0 & 0 & 0 & 0 & 3 & 1,5 \\
\hline Irenomis tarsalis & 1 & 0 & 0 & 0 & 0 & 0 & 1 & 0,5 \\
\hline Dromiciops gliroides & 1 & 1 & 0 & 1 & 0 & 0 & 3 & 1,5 \\
\hline Total & 61 & 96 & 13 & 17 & 8 & 9 & 204 & 100 \\
\hline
\end{tabular}

TABLA 4

Probabilidad de ocurrencia del reservorio de Hantavirus en diferentes hábitats en el área de estudio del Parque Nacional Conguillío, IX Región, 2002

Probability of occurrence of Hantavirus reservoirs in different habitats of Conguillío National Park, 2002

\begin{tabular}{|c|c|c|c|}
\hline \multirow[t]{2}{*}{ Hábitat } & \multirow[t]{2}{*}{ Ocurrencia } & \multicolumn{2}{|c|}{ Superficie } \\
\hline & & (ha) & $(\%)$ \\
\hline Áreas no evaluadas (baja ocurrencia humana) & No evaluado & $2.690,3$ & 14,7 \\
\hline Superficie sin cubierta vegetal & Muy baja & $7.302,6$ & 40,0 \\
\hline Nothofagus antarctica muy ralo con gramíneas & Baja & 111,4 & 0,6 \\
\hline Bosque de A. araucana, $N$. pumilio, Pernettya sp. y $C$. culeu & Baja & $3.279,3$ & 18,0 \\
\hline Matorral de $N$. antarctica & Media baja & 528,4 & 2,9 \\
\hline Bosque de $N$.dombeyi, $N$. pumilio, A. araucana y C. culeu & Media & $1.618,3$ & 8,9 \\
\hline Bosque de $N$.dombeyi- $N$.dombeyi- $N$. alpina-C. culeu & Alta & $2.472,2$ & 13,5 \\
\hline Bosque de A. araucana- $N$. antarctica y Pernettya sp. & Muy alta & 257,4 & 1,4 \\
\hline Total & & $18.259,8$ & 100 \\
\hline
\end{tabular}




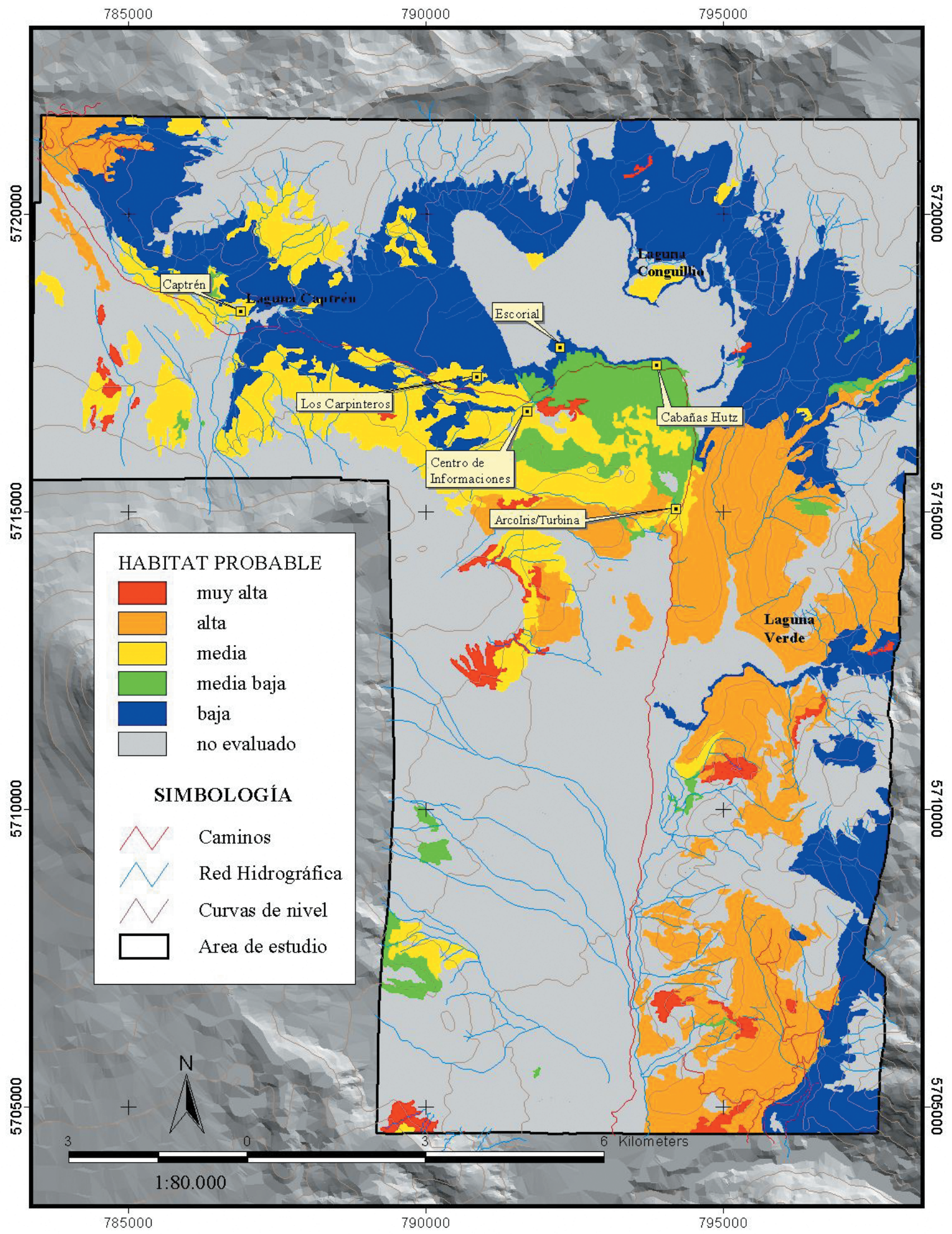

Fig 2: Carta de hábitat probable para roedores en el área de estudio del Parque Nacional Conguillío, 2002.

Map of probable rodent habitat at Conguillio National Park study site, 2002. 
El porcentaje de seropositividad general encontrado en este estudio es alto comparada con otros estudios en Chile (Ortiz et al. 2004, Torres-Pérez et al. 2004) aunque inferior al 5,0 $\%$ encontrado en Valdivia (Padula et al. 2004). Sin embargo, el porcentaje de seroprevalencia en la especie $O$. longicaudatus encontrada en este estudio es superior a la registrada por Padula et al. (2004) de 9,6\%, y el 10,4\% consignado por Torres-Pérez et al. (2004) en Chile y al 8,7 \% registrado por Cantoni et al. (2001) en Argentina, similar al 12,7\% señalado por Toro et al. (1998) en Chile y al 13,5\% encontrada en $O$. flavecens también en Argentina por Suárez et al. (2003) e inferior al $18,2 \%$ encontrado por Larrieu et al. (2004) en Argentina.

En la Tabla 6 se reúnen los antecedentes de seropositividad de los reservorios y los antecedentes epidemiológicos de humanos que se georreferenciaron y emplearon en la carta final. Estos últimos son una fuente de información muy importante. Se georreferenció el lugar del caso humano registrado de $\mathrm{SCPH}$ en el Parque Nacional Conguillío, que correspondió a una mujer de 57 años que desarrollaba labores en el sector denominado, en este estudio, Centro de Informaciones.

De este modo se identificaron tres áreas: (a) área de probabilidad alta de encuentro de un roedor seropositivo, (b) área con registro de caso humano de SHP, (c) área de probabilidad media de encuentro de un roedor seropositivo.

\section{Asentamientos humanos}

Se consideraron las áreas de actividad humana en el Parque Nacional Conguillío como todas las superficies susceptibles de ser utilizadas por seres humanos. Se distinguieron dos áreas con distinto nivel de riesgo, basándose especialmente en el tiempo de permanencia, tipo de exposición (ambiente abierto o cerrado) y actividad desarrollada en el área: la primera,

TABLA 5

Seropositividad de roedores capturados en el Parque Nacional Conguillío, IX Región, 1999-2002

Hantavirus seropositive rodents captured in Conguillio National Park, 1999-2002

\begin{tabular}{lcccc}
\hline Especie & $\begin{array}{c}\text { Número total } \\
\text { de capturas }\end{array}$ & $\begin{array}{c}\text { Ejemplares } \\
\text { seronegativos }\end{array}$ & $\begin{array}{c}\text { Ejemplares } \\
\text { seropositivos }\end{array}$ & $\begin{array}{c}\text { Proporción de } \\
\text { seropositivos }(\%)\end{array}$ \\
\hline Oligoryzomys longicaudatus & 29 & 25 & 4 & 13,8 \\
Abrothrix olivaceus & 39 & 39 & 0 & 0,0 \\
Abrothrix longipilis & 129 & 125 & 4 & 3,1 \\
Loxodontomis micropus & 3 & 3 & 0 & 0 \\
Irenomis tarsalis & 1 & 1 & 0 & 0 \\
Total & 201 & 197 & 8 & 4,0 \\
\hline
\end{tabular}

TABLA 6

Seropositividad de roedores y casos humanos en el Parque Nacional Conguillío, IX Región, 1999-2002

Hantavirus seropositive rodents and a human case at Conguillio National Park, 1999-2002

\begin{tabular}{lcc}
\hline Hábitat & Roedores seropositivos & Casos humanos \\
\hline Nothofagus antarctica muy ralo con gramíneas & No & No \\
Bosque de A. araucana, $N$. pumilio, Pernettya sp. y $C$. culeu & No & No \\
Bosque de $N$. dombeyi, $N$. pumilio, A. araucana y $C$. culeu & No & No \\
Matorral de N. antarctica & No & No \\
Bosque de $N$. obliqua- $N$. dombeyi- $N$. alpina-C. culeu & Sí & No \\
Bosque de A. araucana- $N$. antarctica y Pernettya sp. & Sí & Sí \\
\hline
\end{tabular}


de mayor riesgo, incluye las zonas de picnic dotados de mesones y basureros con permanencia corta ( $<1$ día), áreas de camping dotado de luz, agua y basureros con permanencia media (> 1 día), cabañas con infraestructura completa y permanencia larga (> 1 día) e infraestructura del parque como zona de administración, habitaciones, bodegas, cabañas de guardaparques, con permanencia larga (> $1 \mathrm{mes}$ ); y la segunda los caminos y senderos como áreas de menor riesgo al ser usados solo como tránsito turístico u operativo.

\section{Carta de riesgo}

La clasificación de los niveles de riesgo consideró cuatro niveles. Para el caso de la categoría de muy alto riesgo el modelo consideró: (a) hábitat con registro de caso humano de hantavirus, (b) hábitat de alta ocurrencia del reservorio, (c) área de probabilidad alta de encuentro con un roedor seropositivo y (d) área de tránsito humano cierto (alta probabilidad de presencia humana en movimiento). En la situación inversa, para muy bajo riesgo, consideró: (a) hábitat sin registro de caso humano, (b) hábitat sin probabilidad de ocurrencia del reservorio, (c) ausencia de roedores reservorios seropositivos, y (d) área no susceptible de ser transitada. Los niveles intermedios los calcula el algoritmo matemático del programa, combinando todas las variables en sus diferentes posibilidades. Se programó el modelo con una ponderación igual para cada variable, situación susceptible de modificarse. En la Fig. 3 se muestra el mapa de riesgo, con la información descrita en todo el proceso y considerando las variables con igual ponderación.

La superficie afectada, según los diferentes niveles de riesgo, se muestra en la Tabla 7. En ella se pueden apreciar que solo 5,5 ha pueden ser consideradas de muy alto riesgo en el Parque Nacional Conguillío para el año 2002 y 391,3 ha en alto riesgo, lo que representa un 2,1 $\%$ del total de la superficie estudiada, de la cual más de la mitad presenta un nivel de muy bajo o bajo riesgo (54,1 y $21,8 \%$ respectivamente). Esta información debiera estar incorporada en los planes de manejo del parque estudiado ya que debe ser una variable importante a considerar para localizar las instalaciones, tanto de administración, como de uso turístico (e.g., áreas de camping y cabañas). De hecho, la infraestructura turística está emplazada, precisamente en las zonas de muy alto riesgo, especialmente las zonas de camping. Por esto es que se sugiere reconsiderar estos emplazamientos y relocalizarlos en zonas de bajo riesgo (e.g., bosque de A. araucana, $N$. pumilio, Pernettya sp. y C. culeu, con sotobosque ralo). Proponemos que en todas las áreas silvestres protegidas con presencia de reservorios de hantavirus, con flujos relevantes de turistas y administrativos residentes, se elaboren cartas de riesgos, como las propuestas, y que éstas sean incorporadas en los respectivos planes de manejos de esas unidades.

\section{TABLA 7}

Superficie de territorio de diferentes niveles de riesgo a Hantavirus en el área de estudio del Parque Nacional Conguillío, IX Región, 2002

Areas with different Hantavirus risk levels at Conguillío National Park, 2002

\begin{tabular}{lcc}
\hline Nivel de riesgo & Absoluta (ha) & $\begin{array}{r}\text { Superficie } \\
\text { relativa (\%) }\end{array}$ \\
\hline Muy bajo & $9.876,1$ & 54,1 \\
Bajo & $3.977,5$ & 21,8 \\
Medio & $4.009,4$ & 22,0 \\
Alto & 391,3 & 2,1 \\
Muy alto & 5,5 & 0,0 \\
Total & $18.259,8$ & 100 \\
\hline
\end{tabular}

\section{Variaciones temporales}

Según diferentes factores existirá variación temporal de los mapas de riesgo. Bush et al. (2004) en un análisis espacio-temporal de la distribución de los casos de SCPH en la provincia de Argentina, encontraron que interacción espacio-temporal parece estar relacionada con una estacionalidad fuerte y la existencia de condiciones ecológicas particulares favorables para el reservorio. Las fluctuaciones de las densidades de roedores reservorio son muy variables $(<1$ a $>250$ ind $\mathrm{ha}^{-1}$ año $\left.{ }^{-1}\right)$ tanto en las diferentes estaciones como entre diferentes años (Fulk 1975, Pefaur et al. 1976, Meserve \& Le Boulenge 1987, González et al. 1989, Iriarte et al. 1989, 


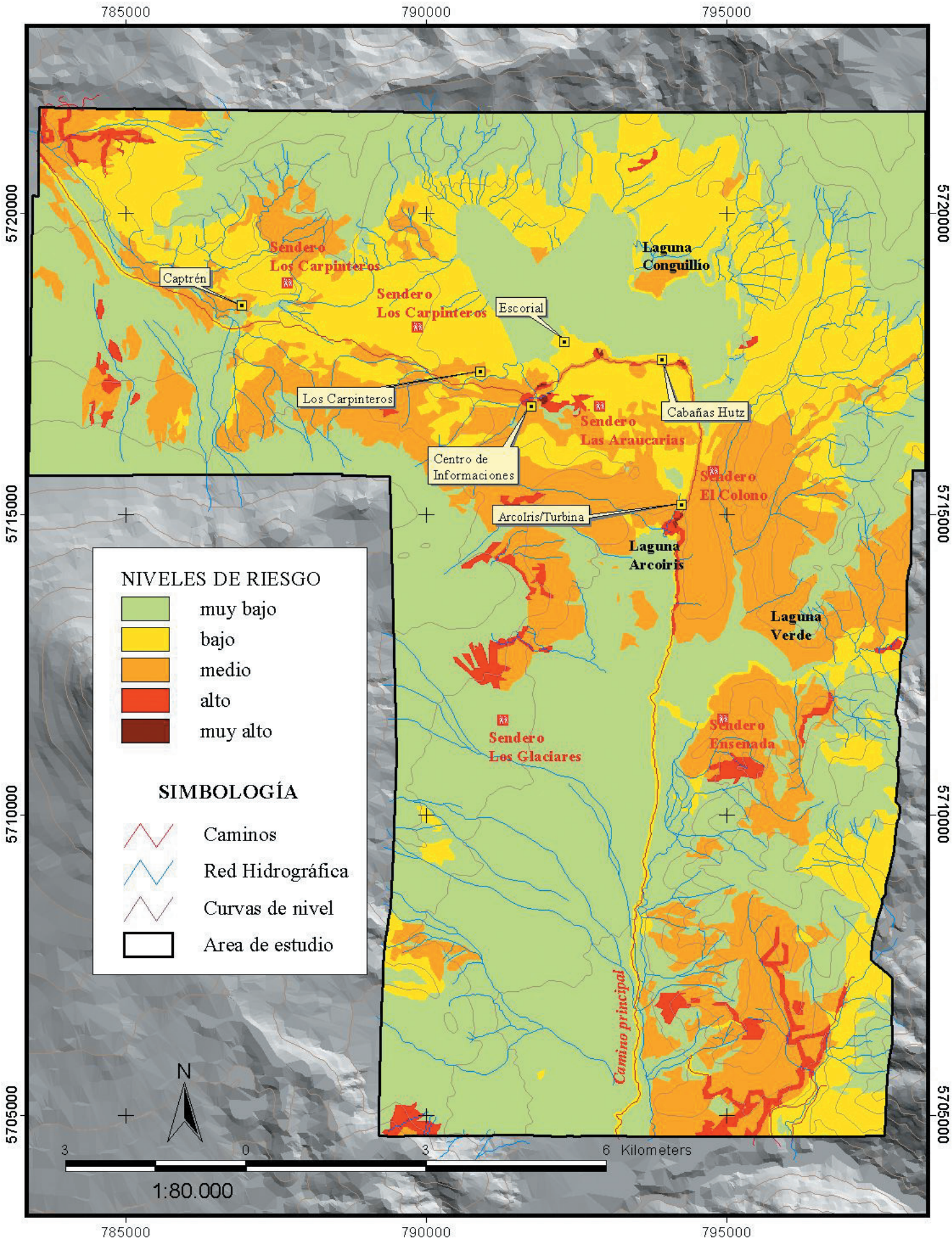

Fig. 3: Carta de riesgo a Hantavirus en el área de estudio del Parque Nacional Conguillío, IX Región, 2002.

Hantavirus risk map at Conguillio National Park study site, 2002. 
Meserve et al. 1991, 1999, Muñoz-Pedreros 1992) (Tabla 8). De esta forma las variaciones ambientales inciden directamente en la dinámica poblacional del reservorio y en consecuencia en los casos de $\mathrm{SCPH}$ registrados, por ejemplo las precipitaciones infrecuentes, el fenómeno del Niño (El Niño Southern Oscillation ENSO), fuerzas estocásticas y el cambio de uso del suelo (González et al. 1989, Engelthaler et al. 1999, Gallardo \& Mercado 1999, González \& Donoso 1999, Lima et al. 1999, Jaksic \& Lima 2003, Murúa et al. 2003). Por este motivo el mapa de riesgo debe estar claramente referido a la estación y año en que fue realizado.

Otro aspecto a considerar es la exposición humana, la que también presentará variaciones, dependiendo de la densidad de la población humana en el momento de elaborar el mapa de riesgo, así como la población expuesta al contacto de aire libre y particularmente la población expuesta en zonas cerradas (Bush et al. 2004). Estas consideraciones serán muy importantes, especialmente en las zonas de uso turístico y/o recreacional como las áreas silvestres protegidas con acceso a visitantes. En un estudio de los visitantes al área de estudio (Correa 2006), en el verano de 2005, el $70 \%$ de los visitantes tenía entre 20 y 45 años de edad, permanecía entre seis y diez días y en un $70 \%$ en zonas de camping. Este tipo de visitantes es una población con características de exposición alta al peligro de contagio con Hantavirus (jóvenes, campistas y con uso intensivo de senderos). La exposición es un factor crucial a tomar en cuenta en las medidas precautorias y preventivas.

Con estas consideraciones se pueden modelar dos escenarios extremos: (a) época de bajo riesgo, de abril a septiembre, ya que se presenta un escenario con bajo número de visitantes, por lo tanto poca población humana expuesta. A esto se puede adicionar un año de baja productividad de semillas y con régimen pluviométrico normal; (b) época de alto riesgo, de octubre a marzo, con alto número de visitantes, por lo tanto numerosa población humana expuesta, en año de alta producción de semilla, con el florecimiento de quila cada 12 14 años (Jaksic \& Lima 2003).

Respecto a la variación de la seropositividad, en algunos lugares como Nuevo México, el $30 \%$ de los roedores han sido seropositivos, sin embargo en muestreos del año siguiente han aparecido negativos. Esto plantea varias interrogantes: ¿el virus se presenta como focos?, ¿el virus se presenta, desaparece, se reactiva y regresa a la población? Por lo tanto, se recomienda el monitoreo de las zonas que han sido descritas con presencia de roedores seropositivos, al menos cada dos años. Es necesario actualizar permanentemente el modelo predictivo en SIG para cada punto con roedores seropositivos, tal como se muestra en la Fig. 4.

TABLA 8

Densidades de roedores registradas en diferentes zonas de Chile

Density of rodents in different areas of Chile

\begin{tabular}{|c|c|c|c|c|c|c|}
\hline Localidad & Hábitat & $\begin{array}{l}\text { Número } \\
\text { trampas }\end{array}$ & $\begin{array}{l}\text { Éxito cap- } \\
\text { tura }(\%)\end{array}$ & $\begin{array}{l}\text { Densidad } \\
\left(\text { ind ha }^{-1} \text { ) }\right.\end{array}$ & Año & Fuente \\
\hline Fray Jorge & Arbustos (44\%) & & & 115 & 1975 & Fulk (1975) \\
\hline Rinconada de Maipú & Arbustos $(70 \%)$ & & & 22 & 1975 & Fulk (1975) \\
\hline Rinconada de Maipú & Arbustos (70\%) & & & 58 & 1989 & Iriarte et al. (1989) \\
\hline Concepción (Burca) & Arbustos (63\%) & 64 & 40 & 97 & $1983-1985$ & Muñoz-Pedreros (1992) \\
\hline Valdivia (San Martín) & Bosque/sotobosque & 144 & & 27 & Varios & $\begin{array}{l}\text { L. González (comunica- } \\
\text { ción personal) }\end{array}$ \\
\hline Valdivia (San Martín) & C. culeu florecida & 144 & & 172 & 1985 & Murúa et al. (1986) \\
\hline Osorno (La Picada) & Bosque/sotobosque & 300 & $18 / 16$ & 36 & $1983-1985$ & $\begin{array}{l}\text { Meserve et al. } \\
(1988,1991)\end{array}$ \\
\hline
\end{tabular}




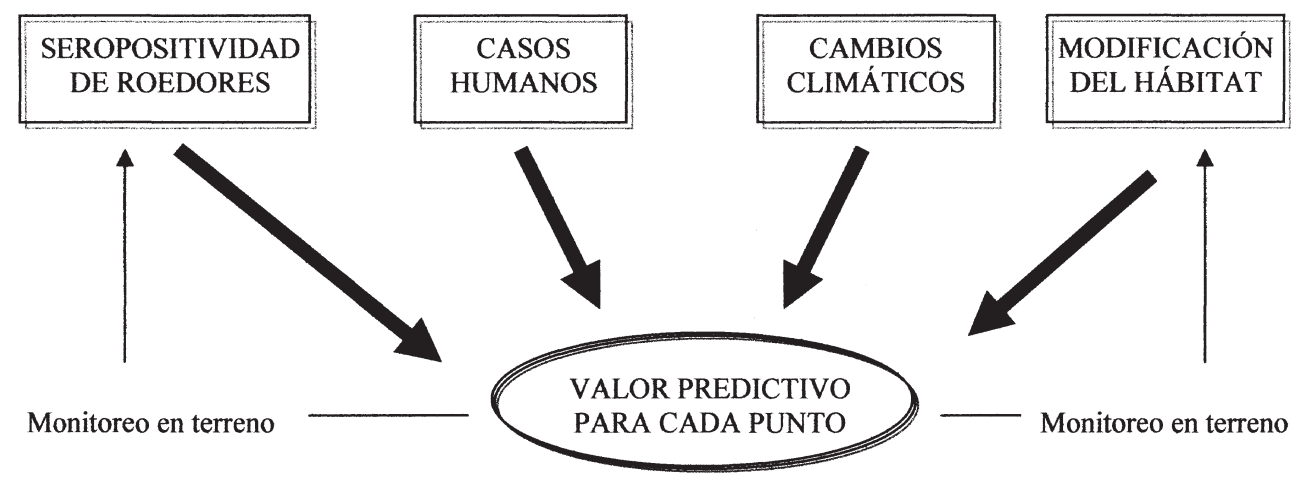

Fig. 4: Diagrama de factores a considerar para la actualización permanente de la información en puntos con roedores seropositivos.

Diagram of the factors to be considered in the permanent data update for seropositive rodents.

\section{AGRADECIMIENTOS}

Los autores agradecen al proyecto FONDEF D99I1 105 ejecutado por la Universidad Austral de Chile y el Centro de Estudios Agrarios y Ambientales. También a Basilio Guiñez de la Corporación Nacional Forestal (IX Región), por las facilidades y ayuda en terreno.

\section{LITERATURA CITADA}

BENOIT I (2004) Antecedentes sobre las áreas silvestres protegidas de la IX Región de la Araucanía. Corporación Nacional Forestal, Chile. 54 pp.

BUSCH M, R CAVIA1, AE CARBAJO, C BELLOMO, S GONZÁLEZ CAPRIA \& P PADULA (2004) Spatial and temporal analysis of the distribution of hantavirus pulmonary syndrome in Buenos Aires Province, and its relation to rodent distribution, agricultural and demographic variables. Tropical Medicine and International Health 9: 508-519.

CANTONI G, P PADULA, G CALDERÓN, J MILLS, E HERRERO, P SANDOVAL, V MARTÍNEZ, N PINI \& E LARRIEU (2001) Seasonal variation in prevalence of antibody to hantaviruses in rodents from southern Argentina. Tropical Medicine and International Health 6: 811-816.

CASTILLO C \& G OSSA (2002) Síndrome pulmonar por Hantavirus Andes en Chile. Revista Chile de Enfermedades Respiratorias 18: 35-46.

CDS/NIH/OPS/OMS (s/f) Métodos para trampeo y muestreo de pequeños mamíferos para estudios virológicos. Organización Panamericana de la Salud/Organización Mundial para la Salud, Santiago, Chile. 66 pp.

CONAF-CONAMA (1999) Catastro y evaluación de recursos vegetacionales nativos de Chile. Proyecto CONAF-CONAMA-BIRF, Santiago, Chile. 87 pp.

CORREA C (2006) Determinación del potencial de uso turístico a través de la evaluación de atractivos turísticos en el Parque Nacional Conguillío, IX
Región. Tesis, Facultad de Recursos Naturales, Universidad Católica de Temuco, Temuco, Chile. $107 \mathrm{pp}$.

COTTAM G, JT CURTISS \& B HALE (1953) Some sampling characteristis of a population of randomly dispersed individuals. Ecology 34: 741-757.

DALMAGRO AD \& EM VIEIRA (2005) Patterns of habitat utilization of small rodents in an area of Araucaria forest in southern Brazil. Austral Ecology 30: 353-362.

DE BLAISE AF \& RE MARTIN (1979) A Manual of Mammalogy. Wn. C. Brown Company Publishers, Dubuque, Iowa, USA. XVI+ $329 \mathrm{pp}$.

ELGH F, A LUNDKVIST, OA ALEXEYEV, H STENLUND, T AVSIC-ZUPANC, B HJELLE, HW LEE, KJ SMITH, R VAINIONPAA, D WIGER, G WADELL \& P JUTO (1997) Serological diagnosis of Hantavirus infections by enzyme -linked immunosorbent assay based on detection of immunoglobulin $\mathrm{G}$ and $\mathrm{M}$ responses to recombinant nucleocapsid proteins of five viral serotypes. Journal of Clinical Microbiology 35: 1122-1130.

ENGELTHALER DM, MOSLEY DG, CHEEK JE, LEVY CE, KK KOMATSU, P ETTESTAD \& T DAVIS (1999) Climatic and environmental patterns associated with Hantavirus pulmonary syndrome, Four Corners Region, United States. Emergent Infection Diseases 5: 87-94.

FULK G (1975) Population ecology of rodents in the semiarid shrub lands of Chile. Occasional Papers Museum, Texas Tech University (USA) 33: 1-40.

GALENO H, E VILLAGRA, J FERNÁNDEZ, E RAMÍREZ \& J MORA (2000) Técnicas diagnósticas de infección humana por hantavirus. Revista Chilena de Infectología 17: 211-215.

GALLARDO MH \& C MERCADO (1999) Mast seeding of bamboo shrubs and mouse outbreaks in southern Chile. Mastozoología Neotropical (Argentina) 6: 103-111.

GLASS GE, TL YATES, JB FINE, TM SHIELDS, JB KENDALL, AG HOPE, CA PARMENTER, CJ PETERS, TG KSIAZEK, C LI, JA PATZI \& JN MILLS (2002) Satellite imagery characterizes local animal reservoir populations of Sin Nombre virus in the southwestern United States. Proceedings of the National Academy of Sciences of the United States of America 99: 16817-16822. 
GONZÁLEZ L, R MURÚA \& C JOFRÉ (1989) The effect of seed availability on population density of Oryzomys in southern Chile. Journal of Mammalogy 70: 401-403

GONZÁLEZ ME \& C DONOSO (1999) Producción de semillas y hojarasca en Chusquea quila (Poaceae: Bambusoideae), posterior a su floración sincrónica en la zona centro-sur de Chile. Revista Chilena de Historia Natural 72: 169-180.

HOFFMANN A (1982) Flora silvestre de Chile. Zona austral. Ediciones Fundación Claudio Gay, Santiago, Chile. 258 pp.

IRIARTE JA, L CONTRERAS \& F JAKSIC (1989) A long-term study of a small mammal assemblage in the central Chilean matorral. Journal of Mammalogy 70: 79-87.

JAKSIC F \& M LIMA (2003) Myths and facts on "ratadas": bamboo blooms, rainfall peaks and mouse outbreaks. Austral Ecology 28: 237-251.

LARRIEU E, E HERRERO, M GARCIA, JL LABANCHI, $S$ MANCINI, P PADULA, G CANTONI, L CAVAGION, E ÁlVAREZ, M BRUNI, S ALBARRACÍN \& O ARELLANO (2003) Seroprevalencia de hantavirus en roedores y casos humanos en el sur de la Argentina. Revista Brasileira de Epidemiologia 6: 68-75.

LIMA M, KEYMER JE \& F JAKSIC (1999) ENSO-driven rainfall variability and delayed density dependence cause rodent outbreaks in western South America: linking demography and population dynamics. American Naturalist 153: 476-491.

MaCARTHUR RH \& J MaCARTHUR (1961) On bird species diversity. Ecology 42: 594-598.

MARTICORENA C \& M QUEZADA (1985) Catálogo de la flora vascular de Chile. Gayana Botánica (Chile) 42: 5-157.

MESERVE PL \& ER LE BOULENGE (1987) Population dynamics and ecology of small mammlas in the northern Chilean searid region. En: Patterson BD \& RM Timm (eds) Studies in Neotropical mammalogy: essay in honor of Philip Hershkovits. Fielddiana: Zoology (New Series) 39: 413-431.

MESERVE PL, BK LANG \& B PATERSON (1988) Trophics relationships of small mammals in a Chilean temperate rainforest. Journal of Mammalogy 69: 721-730.

MESERVE PL, BK LANG, R MURÚA, A MUÑOZPEDREROS \& LA GONZÁLEZ (1991) Characteristic of a terrestrial small mammals assemblage in a temperate rainforest in Chile. Revista Chilena de Historia Natural 64: 157-169.

MESERVE PL, DR MARTÍNEZ, J RAU, R MURÚA, BK LANG \& A MUÑOZ-PEDREROS (1999) Comparative demography and diversity of small mammals in precordilleran temperate rainforest of southern Chile. Journal of Mammalogy 80: 880-890.

MILLS JN (1999) The role of rodents in emerging human disease: examples from hantaviruses and arenaviruses. En: Singleton G, L Hynds, L Herwig \& Z Zhang (eds) Ecologically-based rodent management. ACIAR Monograph Series: 134-160. Canberra, Australia.

MUÑOZ-PEDREROS A (1992) Ecología de la asociación de micromamíferos presentes en un agroecosistema forestal de Chile central. Revista Chilena de Historia Natural 65: 417-42.

MUÑOZ-PEDREROS A, R MURÚA \& L GONZÁLEZ (1990) Nicho ecológico de micromamíferos en un agroecosistema forestal de Chile central. Revista Chilena de Historia Natural 63: 267-277.
MURÚA R, LA GONZÁLEZ \& PL MESERVE (1986) Population ecology of Oryzomys longicaudatus Philippii (Rodentia: Cricetidae) in southern Chile. Journal of Animal Ecology 55: 281-293.

MURÚA R, LA GONZÁLEZ \& M LIMA (2003) Population dynamics of rice rats (a Hantavirus reservoir) in southern Chile: feedback structure and non-linear effects of climatic oscillations. Oikos 102: $137-145$

ORTIZ JC, W VENEGAS, JA SANDOVAL, P CHANDÍA \& F TORRES-PÉREZ (2004) Hantavirus en roedores de la Octava Región de Chile. Revista Chilena de Historia Natural 77: 251-256.

PADULA PJ, A EDELSTEIN, S MIGUEL (1998) Hantavirus pulmonary syndrome outbreak in Argentina: molecular evidence for person to person transmission of Andes virus. Virology 241: 323 330 .

PADULA P, R FIGUEROA, M NAVARRETE, E PIZARRO, R CÁDIZ, C BELLOMO, C JOFRÉ, L ZAROR, E RODRÍGUEZ \& R MURÚA (2004) Transmission study of Andes Hantavirus infection in wild sigmodontine rodents. Journal of Virology 78: 11972-11979

PAVLETIC P (2000a) Hantavirus: su distribución geográfica entre los roedores silvestres de Chile. Revista Chilena de Infectología 17: 186-196.

PAVLETIC P (2000b) Hantavirus: medidas de prevención y manejo ambiental. Revista Chilena de Infectología 17: 270-275.

PEFAUR JE, JL YÁÑEZ \& FM JAKSIC (1979) Biological and environmental aspects of a mouse outbreak in the semiarid region of Chile. Mammalia 43: 313322.

SÁNCHEZ-CORDERO V, A PETERSON, E MARTÍNEZMEYER \& R FLORES (2005) Distribución de roedores reservorios del virus causante del síndrome pulmonar por hantavirus y regiones de posible riesgo en México. Acta Zoológica Mexicana 21: 79-91.

SHCMALJOHN C \& B HJELLE (1997) Hantaviruses: a global disease problem. Emerging Infectious Diseases 3: 95-104.

SOTOMAYOR V \& X AGUILERA (2000) Epidemiología de la infección humana por Hantavirus en Chile. Revista Chilena de Infectología 17: 220-232.

SPOTORNO A, RE PALMA \& J VALLADARES (2000) Biología de roedores reservorios de Hantavirus en Chile. Revista Chilena de Infectología 17: 197-210.

SUÁREZ O, G CUETO, R CAVIA, I GÓMEZ, D BILENCA, A EDELSTEIN, P MARTÍNEZ, S MIGUEL, C BELLOMO, K HODARA, P PADULA \& M BUSCH (2003) Prevalence of infection with Hantavirus in rodent populations of central Argentina. Memorias do Instituto Oswaldo Cruz, Río de Janeiro (Brasil) 98: 727-732

TORO J, JD VEGA, AS KHAN, JN MILLS, P PADULA, W TERRY, Z YADÓN, R VALDERRAMA, BA ELLIS, C PAVLETIC, R CERDA, S ZAKI, S WUN-JU, R MEYER, M TAPIA, C MANSILLA, M BARÓ, JA VERGARA, M CONCHA, G CALDERÓN, D ENRIA, CJ PETERS \& TG KSIAZEK (1998) An outbreak of Hantavirus pulmonary syndrome, Chile, 1997. Emerging Infectious Diseases 4: 687-694.

TORRES-PÉREZ F, J NAVARRETE-DROGUETT, R ALDUNATE, T YATES, G MERTZ, P VIAL, M FERRÉS, P MARQUET \& E PALMA (2004) Peridomestic small mammals associated with confirmed cases of human Hantavirus disease in 
southcentral Chile. American Journal of Tropical Medicine and Hygiene 70: 305-309.

TAPIA M (1997) Caso clínico: síndrome pulmonar por Hantavirus. Revista Chilena de Enfermedades Respiratorias 13: 103-110.
YÁÑEZ J \& A MUÑOZ-PEDREROS (2000) Mamíferos vivientes de Chile. En: Muñoz-Pedreros A \& J Yánez (eds) Mamíferos de Chile: 37-40. CEA Ediciones, Valdivia, Chile.

Editor Asociado: Mauricio Canals

Recibido el 5 de septiembre de 2006; aceptado el 9 de abril de 2007 
